Gestionar: revista de empresa y gobierno

Vol. 1 Núm. 1 (2021)

ISSN: 2810-8264 ISSN-L: 2810-823X

Editada por: Instituto Universitario de Innovación Ciencia y Tecnología Inudi Perú

\title{
Comercio internacional: el Perú como país emergente en el contexto de la COVID-19
}

International trade: Peru as an emerging country in the context of COVID-19

Comércio internacional: Peru como país emergente no contexto da COVID-19

Wilson Sucari ${ }^{1}$

Instituto Universitario de Innovación Ciencia y Tecnología Inudi Perú, Perú

https://orcid.org/0000-0001-5874-0966

DOI: https://doi.org/10.35622/j.rg.2021.01.001

Recibido 30/12/2020/ Aceptado 30/01/2021

RESUMEN. El objetivo fue analizar el comportamiento del Perú en el comercio internacional durante el contexto de la Covid-19. La metodología empleada fue la revisión documental. El análisis se centró en conocer las políticas tomadas por el gobierno peruano frente al comercio internacional en el contexto de la pandemia. Los principales resultados indican que las políticas de gobierno de los últimos años no dieron importancia al aspecto tecnológico con el cual se mueve la economía internacional. Asimismo, el gobierno de 2020-2021 no supo adecuarse a las recomendaciones internacionales como de la Organización Mundial de Comercio y la Organización Mundial de Aduanas; a falta de la implementación de estas políticas el Perú sufrió contracción económica a nivel interno como externo.

PALABRAS CLAVE: Comercio internacional, comercio exterior, comercio mundial, Covid-19, Perú.

ABSTRACT. The objective was to analyze the behavior of Peru in international trade during the context of Covid-19. The methodology used was the documentary review. The analysis focused on knowing the policies taken by the Peruvian government regarding international trade in the context of the pandemic. The main results indicate that government policies in recent years did not give importance to the technological aspect with which the international economy moves. Likewise, the 2020-2021 government did not know how to comply with international recommendations such as the World Trade Organization and the World Customs Organization; In the absence of these policies, Peru suffered an economic contraction both internally and externally.

KEYWORDS: International trade, foreign trade, world trade, covid-19, Peru.

RESUMO. O objetivo foi analisar o comportamento do Peru no comércio internacional durante o contexto da Covid-19. A metodologia utilizada foi a revisão documental. A análise se concentrou em conhecer as políticas do governo peruano em relação ao

${ }^{1}$ Correspondencia: wsucari@inudi.edu.pe 
comércio internacional no contexto da pandemia. Os principais resultados indicam que as politicas governamentais nos últimos anos não deram importância ao aspecto tecnológico com que se move a economia internacional. Da mesma forma, o governo 2020-2021 não soube cumprir recomendações internacionais como a Organização Mundial do Comércio e a Organização Mundial das Alfândegas; na ausência da implementação dessas políticas, o Peru sofreu uma contração econômica interna e externamente.

PALABRAS CLAVE: Comércio internacional, comércio exterior, comércio mundial, Covid-19, Peru.

\section{INTRODUCCIÓN}

El comercio internacional también es conocido como comercio exterior o comercio mundial (Babenko et al., 2019; Han et al., 2018; Jerez, 2011; Urrosolo Muñoz \& Martínez, 2018). Se entiende por comercio internacional al movimiento que tienen los bienes y servicios a través de los distintos países y sus mercados ya sea interno o externo (Ballesteros, 2001).

La práctica del comercio internacional se realiza utilizando divisas y está sujeto a regulaciones adicionales que establecen los participantes en el intercambio y los gobiernos de sus países de origen (Alzate, 2018; Kathuria et al., 2019; Tolstoy et al., 2021). Al realizarse ese hecho económico los países involucrados se benefician mutuamente al posicionar mejor sus productos e ingresar a mercados extranjeros (Emelianov, 2019; Okenna \& Adesanya, 2020).

Los países de América latina no son ajenos al comercio internacional, pues están inmersos en los intercambios económicos, sobre todo como exportador de materiales primas e importador de bienes y servicios de países desarrollados (Gaona, 2019; Infante et al., 2020; Rosales \& Herreros, 2017).

El Perú, en la economía internacional, es considerado como un país emergente que está en vías de desarrollo (Ku Soria, 2017; Mendoza Bellido, 2017). Se caracteriza como exportador de materiales primas e importador de bienes y servicios a diferentes escalas económicas internacionales, sobre todo, de países desarrollados.

No obstante, el desenvolvimiento por la Covid-19 afectó radicalmente las formas de intercambio comercial tradicional. Lo cual tuvo efectos transcendentales en la economía de sus pobladores. 
En ese sentido, este ensayo se planteó como objetivo analizar el comportamiento del Perú en el comercio internacional durante el contexto de la Covid-19.

\section{DESARROLLO}

Como dato fundamental se sabe que la economía peruana cayó en 11,1\% durante 2020, lo cual fue una caída significativa después de 30 años (Forks, 2021). Se sabe también que Perú registró la mayor tasa de personas fallecidas a consecuencia del virus, y fue entre los primeros países con mayor cantidad de personas infectadas a nivel mundial.

En términos económicos, la contracción del Producto Bruto Interno (PBI) de Perú durante el 2020 fue superior a la de los demás países de América Latina (Vega, 2020).

Para Guadalupe (2021) la pandemia del COVID-19 “(...) puso en evidencia falencias en la logística operativa del comercio exterior peruano relacionadas con el uso no articulado de instrumentos tecnológicos, situación que no permitía la interacción transversal y eficiente de los distintos eslabones de dicha cadena" (p. 6).

Frente a esta realidad, podemos afirmar que las políticas empleadas por los gobiernos de los últimos años no visionaron la dinámica social del mundo. Por ende, el país no estaba involucrado significativamente en el uso de la tecnología, con la cual se movió el comercio internacional.

Otro de los aspectos a considerar para desenvolvimiento del comercio internacional fue el seguir las reconvenciones de la Organización Mundial de Aduanas (OMA); a continuación, se presenta la tabla de recomendaciones: 


\section{Figura 1:}

Recomendaciones de la Organización Mundial de Aduanas (OMA) para las políticas de gobierno frente a la COVID-19

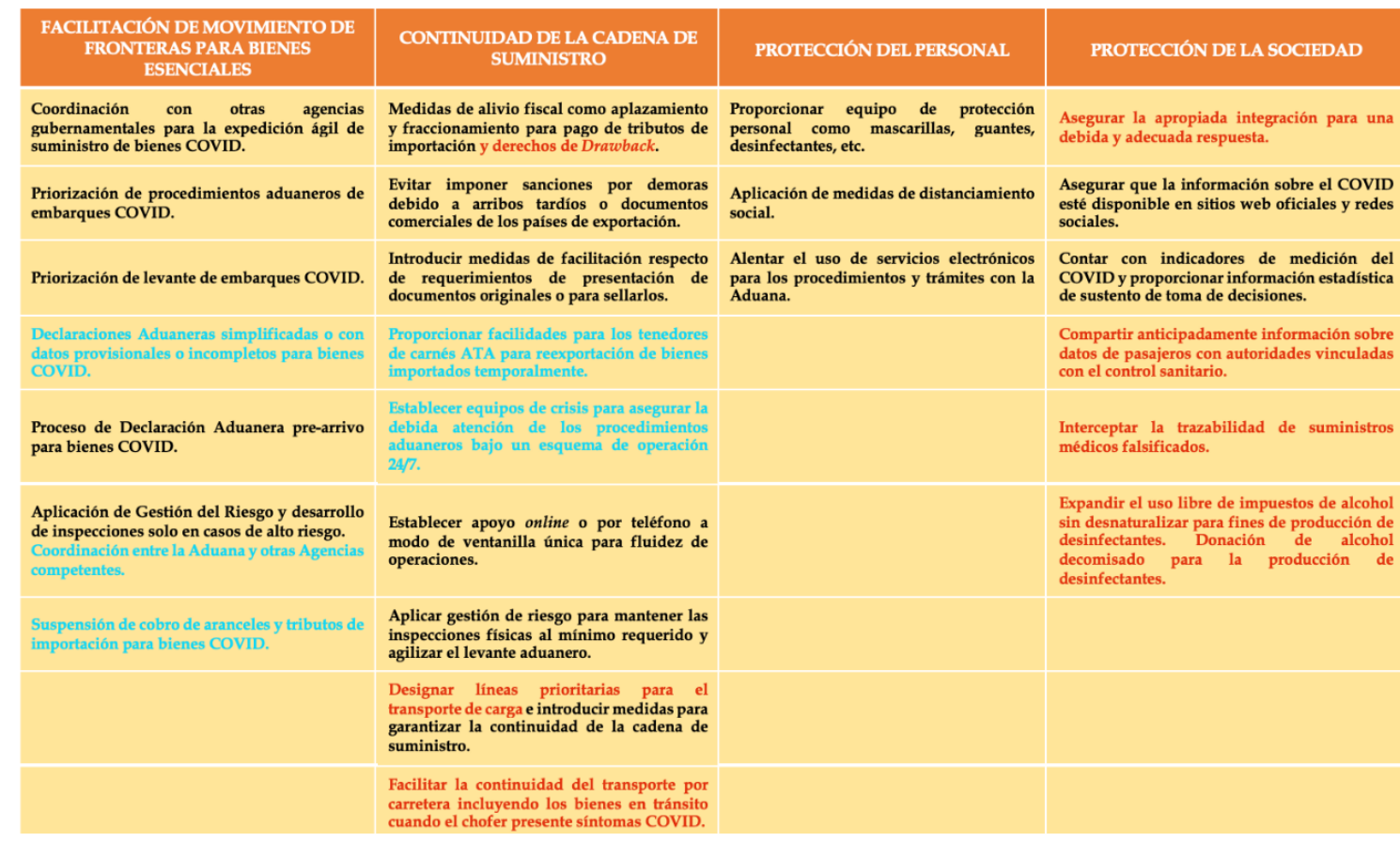

Fuente: Guadalupe (2021).

Nota. Color negro está referido a medidas que ya habían sido operativizadas en el Perú antes de la pandemia por el COVID-19 / Color celeste está referido a las medidas operativizadas reforzadas en el Perú con ocasión de la pandemia por el COVID-19. / Color rojo está referida a las medidas no operativizadas en el Perú.

Pues estas recomendaciones debieran ser asumidas por el gobierno del Perú para que el comercio internacional siga su curso a pesar de la pandemia.

A modo de discusión podemos decir que definitivamente las nuevas tecnologías están facilitando en gran medida la operatividad del comercio exterior. Los sistemas informáticos y de gestión permiten hacer seguimiento de los envíos, acceder a todos los datos relevantes de un contenedor o grupaje en destino, así como compartir y administrar la documentación necesaria de forma fácil.

El comercio internacional también se está viendo fomentado por la mayor facilidad de encontrar clientes en el exterior, gracias al mayor alcance de los portales corporativos en Internet. 


\section{CONCLUSIONES}

En conclusión, el comercio internacional en la actualidad requiere el uso de los recursos tecnológicos, por su operatividad y automatización.

En el contexto de la Covid-19, el Perú no supo adaptarse a las recomendaciones de los organismos internacionales, lo que trajo como consecuencia la recesión económica más alta de las últimas tres décadas.

Se hace necesario una transformación tecnológica, para que el país se adapte a la nueva forma de comercialización digital, de manera que se contribuya al crecimiento de la economía.

\section{REFERENCIAS}

Alzate, D. (2018). Efecto divisa en el comercio internacional: mecanismos para minimizar el riesgo. Universidad de San Buenaventura Colombia.

Babenko, V., Kulczyk, Z., Perevosova, I., Syniavska, O., \& Davydova, O. (2019). Factors of the development of international e-commerce under the conditions of globalization. SHS Web of Conferences, 65, 04016. https:/ / doi.org/10.1051/shsconf/20196504016

Ballesteros, A. (2001). Comercio internacional: teoría y práctica. EDITUM.

Emelianov, E. V. (2019). Public opinion in Russia, USA, other countries on risks and benefits of international trade. International Trade and Trade Policy, 1, 95-104. https:/ / doi.org/10.21686/2410-7395-2019-1-95-104

Forks, J. (2021, febrero). Crisis por coronavirus: La economía en Perú cayó un 11\% en 2020, el mayor retroceso en 30 años | Economía | EL PAÍS. El País.

Gaona, E. (2019). Modelo Primario-Exportador en México y en América Latina, 1870-1930. Boletín Científico de las Ciencias Económico Administrativas del ICEA, $7(14), \quad 1-5$. https://doi.org/10.29057/icea.v7i14.3900

Guadalupe, J. (2021). COVID-19, comercio exterior peruano y lecciones por aprender. Forseti: Revista de Derecho, 9(13), 6-20. https://doi.org/10.21678/forseti.v9i13.1477

Han, H., Xu, H., \& Chen, H. (2018). Social commerce: A systematic review and data synthesis. Electronic Commerce Research and Applications, 30, 3850. https:// doi.org/10.1016/j.elerap.2018.05.005 
Infante, J., Urrego, A., \& Tello, E. (2020). Las venas abiertas de América Latina en la era del Antropoceno: Un estudio biofísico del comercio exterior (1900-2016). Diálogos Revista Electrónica, 21(2), 177-214. https://doi.org/10.15517/dre.v21i2.39736

Jerez, J. L. (2011). Comercio internacional. Esic editorial.

Kathuria, Sanjay, Grover, A., Perego, V. M. E., Mattoo, A., \& Banerjee, P. (2019). Understanding E-Commerce. En Unleashing E-Commerce for South Asian Integration (pp. 7-14). The World Bank. https:/ / doi.org/10.1596/978-1-4648-1519-5_ch1

Ku Soria, P. (2017). PERÚ COMO PRIMER EXPORTADOR DE QUINUA A $\begin{array}{llll}\text { NIVEL MUNDIAL. Quipukamayoc, 25(47), } & 75 .\end{array}$ https://doi.org/10.15381/quipu.v25i47.13805

Mendoza Bellido, W. (2017). La macroeconomía de la flotación sucia en una economía primario exportadora: el caso del Perú. Economía, 40(79), 105132. https://doi.org/10.18800/economia.201701.004

Okenna, N. P., \& Adesanya, B. M. (2020). International Trade and the Economies of Developing Countries. American International Journal of Multidisciplinary Scientific Research, 6(2), 31-39. https://doi.org/10.46281/aijmsr.v6i2.747

Rosales, O., \& Herreros, S. (2017). Desafíos de la competitividad exportadora en América Latina y el Caribe. Estudios internacionales (Santiago), 49, 125141. https://doi.org/http:/ / dx.doi.org/10.5354/0719-3769.2017.47536

Tolstoy, D., Nordman, E. R., Hånell, S. M., \& Özbek, N. (2021). The development of international e-commerce in retail SMEs: An effectuation perspective. Journal of World Business, 56(3), 101165. https://doi.org/10.1016/j.jwb.2020.101165

Urrosolo Muñoz, M., \& Martínez, E. (2018). Gestión administrativa del comercio internacional. Ediciones Paraninfo, SA.

Vega, J. (2020). Crónica de la economía peruana en tiempos de pandemia. Publicaciones PUCP; Pontificia Universidad Católica del Perú. https://doi.org/10.18800/2079-8474.0495 